\title{
Comparison of the Radiation Dose from Cone Beam Computed Tomography and Multidetector Computed Tomography in Examinations of the Hand
}

\section{Vergleich der Strahlendosis von Cone-Beam Computertomografie und Multidetektor Computertomografie in Untersuchungen der Hand}

Authors

Affiliations
J. Neubauer ${ }^{1}$, C. Neubauer ${ }^{1}$, A. Gerstmair ${ }^{1}$, T. Krauss ${ }^{1}$, K. Reising ${ }^{2}$, H. Zajonc ${ }^{3}$, E. Kotter ${ }^{1}$, M. Langer ${ }^{1}$, M. Fiebich ${ }^{4}$, J. Voigt ${ }^{4}$

Department of Radiology, University Medical Center Freiburg, Germany

2 Department of Orthopedics and Trauma Surgery, University Medical Center Freiburg, Germany

${ }^{3}$ Department of Plastic and Hand Surgery, University Medical Center Freiburg, Germany

${ }^{4}$ Institut of Medical Physics and Radiation Protection, University of Applied Sciences, Giessen, Germany

\author{
Key words \\ Q hand \\ - CT \\ - radiation safety \\ - cone beam computed \\ tomography \\ - CBCT \\ low dose
}

\section{Abstract \\ $\nabla$}

Purpose: Comparison of radiation dose of cone beam computed tomography (CBCT) and multidetector computed tomography (MDCT) in examinations of the hand.

Materials and Methods: Dose calculations were carried out by means of Monte Carlo simulations in MDCT and CBCT. A corpse hand was examined in a 320-row MDCT scanner and a dedicated extremities CBCT scanner with standard protocols and multiple lowdose protocols. The image quality of the examinations was evaluated by 5 investigators using a Likert scale from 1 (very good) to 5 (very poor) regarding depiction of cortical bone, cancellous bone, joint surfaces, soft tissues and artifacts. For a sum of ratings of all structures $<50$ a good overall image quality was expected. The studies with at least good overall image quality were compared with respect to the dose.

Results: The dose of the standard examination was 13.21 (12.96 to $13.46 \mathrm{CI}$ ) $\mathrm{mGy}$ in MDCT and 7.15 (6.99 to $7.30 \mathrm{CI}$ ) $\mathrm{mGy}$ in CBCT. The lowest dose in a study with good overall image quality was 4.54 (4.43 to $4.64 \mathrm{CI}$ ) $\mathrm{mGy}$ in MDCT and 5.72 (5.59 to $5.85 \mathrm{CI}$ ) $\mathrm{mGy}$ in CBCT. Conclusion: Although the dose of the standard protocols in the CBCT is lower than in the MDCT, the MDCT can realize a good overall image quality at a lower dose than the CBCT. Dose optimization of CT examination protocols for the hand is useful in both modalities, the MDCT has an even greater potential for optimization.

Key points:

- Low dose examinations of the hand are feasible in CBCT and MDCT.

- In default settings CBCT has a lower dose than MDCT.
- MDCT enables a good image quality at a lower dose than CBCT.

\section{Citation Format:}

- Neubauer J, Neubauer C, Gerstmair A et al. Comparison of the Radiation Dose from Cone Beam Computed Tomography and Multidetector Computed Tomography in Examinations of the Hand. Fortschr Röntgenstr 2016; 188: 488-493

\section{Zusammenfassung}

$\nabla$

Ziel: Vergleich der Strahlendosis von Cone-Beam Computertomografie (CBCT) und MultidetektorComputertomografie (MDCT) bei Untersuchungen der Hand.

Material und Methoden: Dosisbestimmungen wurden mittels Monte Carlo-Simulationen an MDCT und $\mathrm{CBCT}$ durchgeführt. Eine Leichenhand wurde in einem 320-Zeilen MDCT-Scanner und einem für die Bildgebung der Extremitäten ausgerichteten CBCT-Scanner mit Standardprotokollen und multiplen Niedrigdosisprotokollen untersucht. Die Bildqualität der Untersuchungen wurde von 5 Untersuchern mittels einer Likert-Skala von 1 (sehr gut) bis 5 (sehr schlecht) bezüglich der Abbildung von Kortikalis, Spongiosa, Gelenkflächen, Weichteilen und Artefakten beurteilt. Bei einer Summe der Bewertungen aller Strukturen $<50$ wurde eine gute Gesamtbildqualität angenommen. Die Untersuchungen mit mindestens guter Gesamtbildqualität wurden bezüglich der Dosis verglichen.

Ergebnisse: Die Dosis der Standarduntersuchung war 13,21 (KI 12,96-13,46) mGy im MDCT und 7,15 (KI 6,99-7,30) mGy im CBCT. Die niedrigste Dosis in einer Untersuchung mit guter Gesamtbildqualität war 4,54 (KI 4,43-4,64) mGy im MDCT und 5,72 (KI 5,59 - 5,85) mGy im CBCT.

Schlussfolgerung: Obwohl die Dosis der Standardprotokolle in der CBCT niedriger ist als in der MDCT, kann die MDCT eine gute Gesamtbildquali- 
tät bei geringerer Dosis als die CBCT realisieren. Die Dosisoptimierung der CT-Untersuchungsprotokolle für die Hand ist in beiden Modalitäten sinnvoll, wobei die MDCT ein größeres Optimierungspotenzial aufweist.

\section{Introduction}

\section{$\nabla$}

Cone Beam CT Scanners (CBCT) are compact flat detector computed tomography systems that have become well established in craniofacial diagnostics over the last 15 years [1 - 3]. For the past few years these systems have been sold commercially for imaging of the extremities $[4,5]$. The primary difference between the CBCT and modern multidetector computed tomography systems (MDCT) is the detector [6]. The flat detector enables a higher spatial resolution [7], but has a lower dynamic range and is more susceptible to scatter radiation; in contrast to multi-slice detectors in MDCT it does not have a scatter radiation grid [8]. For this reason, as well as the significantly higher generator power, a MDCT provides better soft tissue contrast [9].

There is differing information in the literature with respect to the dose associated with craniofacial examinations. Many studies present a significantly lower dose in CBCT protocols compared to MDCT protocols [10 - 12].

When the two modalities are compared at the same dose however, MDCT provides better contrast resolution [6, 9], and to some extent better spatial resolution as well [6].

Due to the smaller volume of the examination object, examinations of the hand produce less scatter radiation compared to cranial examinations. As this could represent a benefit when using the flat detector, we asked whether the CВСТ was capable of performing high-quality examinations of the hand at a lower dose than the MDCT.

\section{Materials and Methods}

$\nabla$

\section{Phantom}

The examinations were performed on the cadaver hand from a body donor. This meant that an additional vote by the Ethics Commission was unnecessary.

\section{Dose calculations}

Calculating the effective dose for examinations on the extremities based on equivalent doses is difficult, because there are no conversion factors for CT examinations of the extremities, and, in particular none for CBCT examinations. The concept for fluoroscopic examinations [13] may be applied, but in this case there is only information regarding standard projections (a.p., p.a., lat.). Thus, the concept may be insufficient for CBCT dose calculations. However, to make a reliable assertion regarding the applied dose for examinations of the hand, we determined radiation exposure using Monte Carlo simulation. At the beginning of this study, models of the systems to be observed in this regard were created, validated, and calibrated. The uncertainties of the simulation with respect to validation measurements in a Standard Computed Tomography Dose Index (CTDI) head phantom were less than $5 \%$.

The volumes examined contained no organs at risk that had to be included in the calculation of effective dose. For this reason, the absorbed energy dose in individual anatomical structures of the hand was used for the comparison. This results in dose values in $\mathrm{mGy}$ instead of $\mathrm{mSv}$, which are not comparable with specifications of effective dose. They apply only to the comparison of the two scanners examined. The absorbed dose was calculated in the arm of the ICRP male phantom [14], which was extracted from the data set to simplify matters. The simulations for the respective $\mathrm{KV}$ and mAs combination were performed using GMCTdospp software [9].

\section{CT examinations \\ MDCT}

A 320 detector row MDCT (Aquilion One, Toshiba, Otawarashi, Japan) was used. Images were acquired with a 0.5 second $180^{\circ}$ rotation and without table movement. The examinations were performed in standard dose (120 KV und $40 \mathrm{mAs}$ ) and every possible combination of lower $\mathrm{KV}$ and mAs; that is, in all combinations of 80 to $120 \mathrm{KV}(80 \mathrm{KV}, 100$ $\mathrm{KV}, 120 \mathrm{KV}$ ) and 5 to $40 \mathrm{mAs}$ ( $5 \mathrm{mAs}, 7 \mathrm{mAs}, 10 \mathrm{mAs}, 15 \mathrm{mAs}$, $20 \mathrm{mAs}, 30 \mathrm{mAs}, 40 \mathrm{mAs}$ ). In total, examinations were performed using 21 different dose settings. Axial slices were reconstructed with a slice thickness and slice spacing of $0.2 \mathrm{~mm}$ each. The image matrix was $512 \times 512$ pixels.

\section{CBCT}

A CBCT scanner designed for imaging of the extremities (Verity, Planmed, Helsinki, Finland) was used. Images were acquired through 300 projections during an 18 -second $210^{\circ}$ rotation without table movement. The examinations were performed in standard dose (90 KV und $36 \mathrm{mAs}$ ) and every possible combination of lower $\mathrm{KV}$ and $\mathrm{mAs}$; that is, in all combinations of 80 to $90 \mathrm{KV}$ ( $80 \mathrm{KV}, 84 \mathrm{KV}, 88 \mathrm{KV}, 90 \mathrm{KV})$ and 12 to $36 \mathrm{mAs}$ (12 mAs, $18 \mathrm{mAs}, 24 \mathrm{mAs}, 30 \mathrm{mAs}$, $36 \mathrm{mAs}$ ). In total, examinations were performed using 20 different dose settings. Axial slices were reconstructed with a slice thickness and slice spacing of $0.2 \mathrm{~mm}$ each. The image matrix was $801 \times 801$ pixels.

\section{Semiquantitative image quality determination}

The images were sent to the PACS (AGFA Impax 6, Agfa, Mortsel Belgium). The images were assessed under standard conditions in accordance with DICOM 14 [15]. Five raters, unaware of the modality (three radiologists with 1 , 3 , and 5 years experience, and two trauma surgeons with 10 and 18 years experience), performed the evaluations independently of one another. We used numerous image quality descriptors for the semiquantitative image quality determination: cortical bone, cancellous bone, articular surfaces, soft tissue, and artifacts. The raters evaluated the depiction of the cortical bone, cancellous bone, articular surfaces, and soft tissue in the examinations using a Likert scale of 1 (very good), 2 (good), 3 (acceptable), 4 (poor), and 5 (very poor), as suggested by Dehmeri [16]. In addition, the extent of artifacts was evaluated using a Likert scale of 1 (none), 2 (slight, with no impact on diagnosis), 3 (moderate, with no impact on diagnosis), 4 (significant, with impact on diagnosis), and 5 (very significant, with impact on diagnosis). The sum of evaluations for the depiction of cortical bone, cancellous bone, articular surfaces, and soft tissue were added up individually for each examination, and plotted against the dose. If the sum for an individual structure 
gave a value $<10$, good image quality was assumed regarding depiction of this structure. The sum of ratings for the artifacts were added up individually for each examination, and plotted against the dose. A sum of these ratings by the 5 raters of $<10$ each was assumed to denote non-relevant artifacts.

To analyze overall image quality, all 25 evaluations of an examination (5 raters, each with 5 quality descriptors) were summed and plotted against the dose. A sum $<50$ was assumed to denote good overall image quality.

\section{Statistics}

Pearson Correlation was used to analyze the correlation between the ratings and the experience level of the raters [17]. The correlation between the ratings of all 5 raters was analyzed using Kendall's W, with correction for ties [18]. $\mathrm{P}=0.05$ was accepted as the significance level. A Bonferroni P-value correction was performed to prevent familywise error rates [19]. The statistical analysis took place in $\mathrm{R}$ (version 3.0.3).

\section{Results}

$\nabla$

The calculated average dose of the hand for the various settings ranged from 1.14 (CI $1.11-1.17$ ) $\mathrm{mGy}$ to $34.36(\mathrm{CI}$ 33.50 - 35.22) $\mathrm{mGy}$ in MDCT, and from 1.76 (CI 1.73 - 1.79) mGy to 7.15 (CI $6.99-7.30$ ) $\mathrm{mGy}$ in CBCT. The dose for the standard examination in MDCT was higher, at 13.21 (CI 12.96-13.46) $\mathrm{mGy}$, than in CBCT, which was 7.15 (CI 6.99 - 7.31) mGy (॰ Fig. 1A).

MDCT achieved good depiction of the cancellous bone and imaging without relevant artifacts at a lower dose than was the case with CBCT ( $\bullet$ Table $1, \bullet$ Fig. 2,3 ). In contrast, CBCT achieved good depiction of the cortical bone and articular surfaces at a lower dose than was the case with MDCT ( $\bullet$ Table 1, $\bullet$ Fig. 2). Neither modality achieved good depiction of soft tissue ( $\bullet$ Fig. 2 ).
Among all examinations with good overall image quality (sum of all evaluations <50), MDCT achieved the lowest dose, 4.54 (CI $4.43-4.64) \mathrm{mGy}$ with an application of 100 $\mathrm{KV}$ and $20 \mathrm{mAs}$, and CBCT achieved a minimum dose of 5.72 (CI 5.59-5.85) mGy with an application of $90 \mathrm{KV}$ and $30 \mathrm{mAs}(\bullet$ Fig. 1B, 4).

The experience of the raters correlated significantly with a poorer assessment of the MDCT $(\mathrm{P}=0.004)$. In contrast, with CBCT there was no correlation between the experience level and the assessments by the raters $(P=0.59)$. The correlation among all raters equaled $0.43(\mathrm{P}<0.001)$.

\section{Conclusion}

Although the dose of the standard protocols in the CBCT is lower than in the MDCT, the MDCT can realize a good overall image quality at a lower dose than the CBCT. Dose optimization of CT examination protocols for the hand is useful in both modalities, the MDCT has an even greater potential for optimization.

Table 1 Minimal dose of MDCT and CBCT resulting in a good image quality regarding depiction of cortical bone, cancellous bone, articular surfaces, soft tissues and non-relevant artifacts.

\begin{tabular}{|lll|}
\hline & MDCT & CBCT \\
\hline & $\begin{array}{l}\text { dose in mGy } \\
\text { (confidence interval) }\end{array}$ & $\begin{array}{l}\text { dose in mGy } \\
\text { (confidence interval) }\end{array}$ \\
\hline cortical bone & 4.95 & 4.1 \\
\hline cancellous bone & $(4.86-5.04)$ & $(4.03-4.19)$ \\
\hline articular surfaces & 2.31 & 5.42 \\
\hline soft tissue & $(2.27-2.35)$ & $(5.30-5.54)$ \\
\hline non-relevant & none & 2.17 \\
artifacts & 1.14 & $(2.12-2.22)$ \\
\hline
\end{tabular}

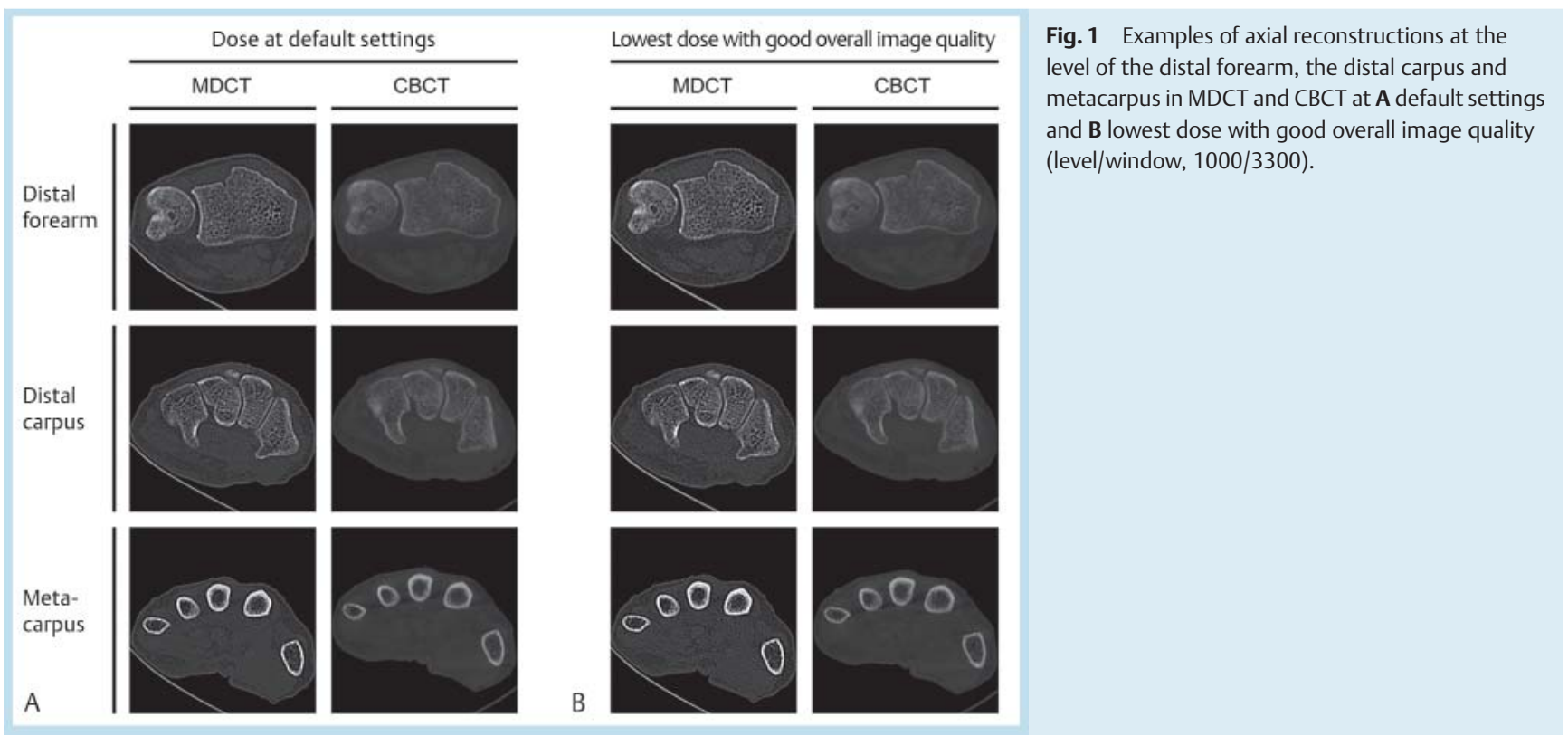



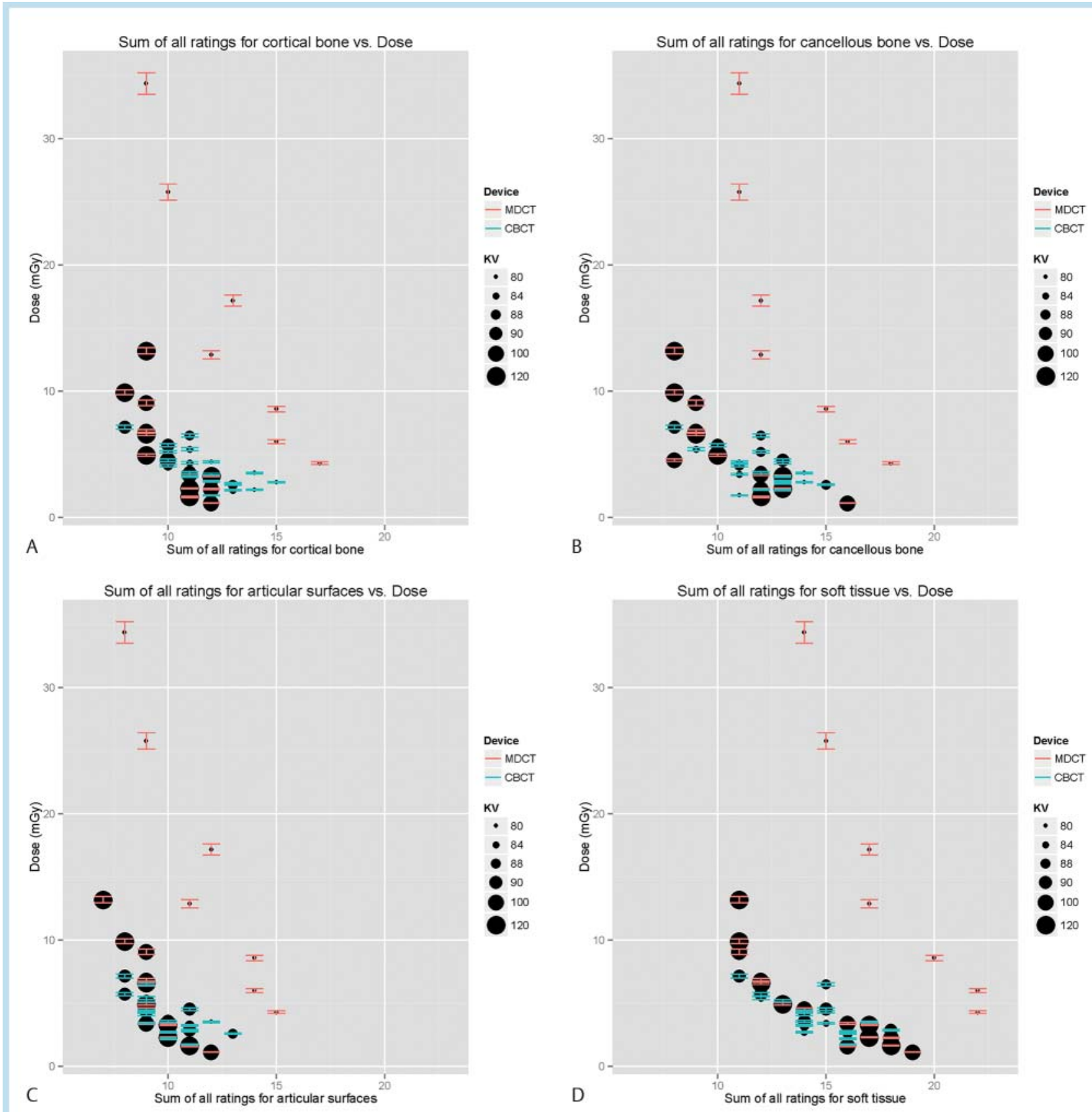

Fig. 2 Sum of all ratings for $\mathbf{A}$ cortical bone, $\mathbf{B}$ cancellous bone, $\mathbf{C}$ articular surfaces and $\mathbf{D}$ soft tissues plotted against the dose for each examination.

\section{Discussion \\ $\nabla$}

In this study on the comparison of the dose between MDCT and CBCT for examinations of the hand, we were able to show that MDCT requires less dose to perform an examination with good overall image quality. CBCT has a lower dose when comparing standard settings.

Good overall image quality using low-dose protocols was possible on both units. This shows that dose optimization for the protocols makes sense for both modalities, although there is greater potential for optimization in MDCT. An advantage for the MDCT can be seen only with reduced-dose imaging protocols.

We used semiquantitative evaluation by raters to evaluate image quality, as this enabled us to analyze the image quality relevant in the clinical routine. Overall image quality was determined using a number of descriptors, most of which referred to bones. This correlates with the clinical requirements regarding non-contrast CT examination of the wrist $[16,20]$. We selected raters from various disciplines and different levels of experience in order to better represent the various user groups. This could also be the most important reason why the correlation between the raters was only moderate. This suspicion is confirmed by the fact that the experienced evaluators judged MDCT significantly worse. Apparently the experienced evaluators were used to the standard image quality of MDCT and evaluated the low dose protocols accordingly worse. That this did not happen with CBCT as well 


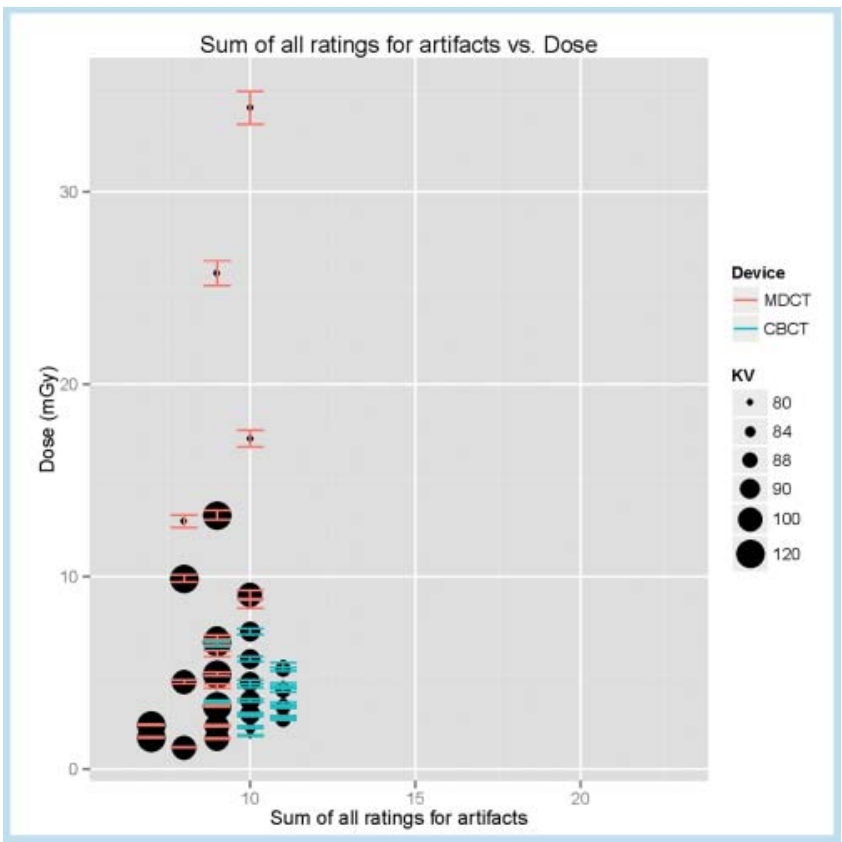

Fig. 3 Sum of all ratings for artifacts plotted against the dose for each examination.

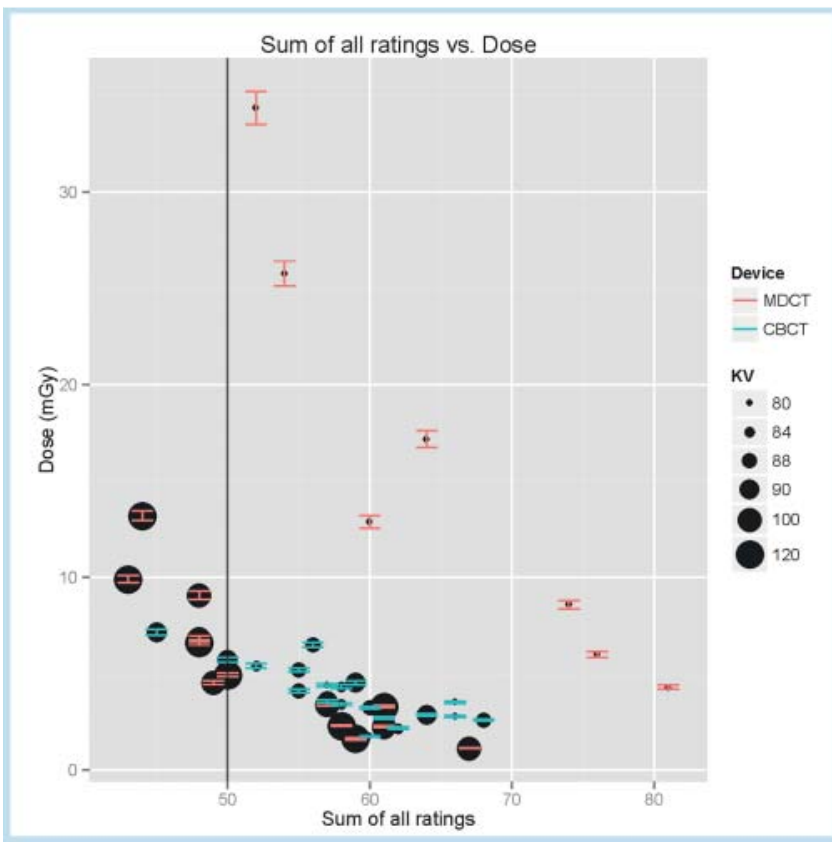

Fig. 4 Sum of all ratings plotted against the dose for each examination.

was not a surprise, as all of the evaluators had limited experience, approximately 6 months, with CBCT.

For the application of CBCT in the head area and extremities, multiple studies indicate a lower radiation exposure than with MDCT $[11,12,21,22]$. However, these studies failed to apply the dedicated dose optimization in MDCT protocols, which limits the comparability of both modalities. In our study we were able to show that dose optimization of the examination protocol is both possible and crucial, particularly for MDCT. We were able to show that it is possible to realize good overall image quality in MDCT at a lower dose than in CBCT. These data are supported by previously pub- lished studies that determined, when comparing MDCT and CBCT at the same dose, better contrast resolution in MDCT and an equivalent display of fractures $[6,9,23]$.

Similar to our results, in examinations of the midface Hoffmann et. al were able to show that diagnostically usable scans in MDCT were possible at a dose lower than CBCT in some cases, depending on the device used [24]. The diverging results between the different studies can be explained by the fact that CBCT scanners in some cases differ significantly with respect to image quality and radiation dose. In addition, different dose settings were used in these studies $[6,24,25]$.

Looking individually at the image quality descriptors in our study, it is clear that the greatest differences in quality for both modalities were in the area of artifacts; MDCT presented significantly fewer artifacts. The susceptibility of CBCT to artifacts has already been documented and is due in large part to technical reasons [26]. The two modalities are relatively close to one another for the other analyzed structures, although the CBCT enables a good representation of cortical bone and articular surfaces at a lower dose than MDCT. This is because CBCT has better spatial resolution than MDCT, enabling high-contrast structures such as bone to be better presented. For cancellous bone, MDCT enables a good depiction at a lower dose than CBCT. This is surprising given that the literature describes a superior depiction of cancellous bone using CBCT. However, we analyzed low-dose protocols in our study where, due to the reduction in radiation dose, image noise rises quadratically to dose reduction. It can be assumed that the iterative reconstruction technique in MDCT better compensates for the increase in image noise. There is no iterative reconstruction technique currently available for the model of $\mathrm{CBCT}$ scanner used.

It was notable that the dose values of the $80 \mathrm{KV}$ MDCT protocols in some cases were significantly higher than those of the other examination protocols. The dose increase at a reduction in ray tube voltage to $80 \mathrm{KV}$ in the MDCT can be explained by the corresponding low-energy spectrum of the $\mathrm{X}$-ray beams, which deposit more dose in the examination object. The significantly stronger prefiltering of the $\mathrm{CBCT}$ scanner explains why the same effect is not observed with CBCT.

Although a study with good overall image quality was conducted in lowest dose by MDCT in our experimental study, a good depiction of cortical bone and joint surfaces was possible with lower dose in the CBCT. СВCT also has other advantages, such as higher spatial resolution. The CBCT has a simpler technical design [1, 27], which may mean lower production and maintenance costs [28]. In addition, because of its limited examination spectrum operation of the CBCT scanners is generally easier and faster to learn. However, additional studies are necessary to confirm this. In our opinion, both modalities are suited to imaging the hand, but dose optimization in the protocols for both modalities is strongly recommended.

\section{Limitations}

The results of our experimental study are limited to the two scanners used. Cadaver hands were used as examination objects, as we could not justify multiple examinations on 
patients. An analysis of sensitivity and specificity of the modalities for specific pathophysiological changes (such as fractures or erosive changes) and for motion artifacts therefore was not possible.

\section{Clinical relevance of the study}

Both MDCT and CBCT achieve good image quality in dose-optimized protocols. Although the CBCT applies a lower dose in the standard setting, the lowest dose in an examination with good overall image quality could be realized with the MDCT. Adapting the examination protocols in both modalities therefore appears to be absolutely necessary, although there is greater potential for optimization with MDCT.

\section{References}

1 Scarfe WC, Farman AG. What is Cone-Beam CT and How Does it Work? Dent Clin North Am 2008; 52: 707 - 730

2 Tyndall DA, Price JB, Tetradis S et al. Position statement of the American Academy of Oral and Maxillofacial Radiology on selection criteria for the use of radiology in dental implantology with emphasis on cone beam computed tomography. Oral Surg Oral Med Oral Pathol Oral Radiol 2012; 113: 817-826

3 Kapila S, Conley RS, Harrell WE. The current status of cone beam computed tomography imaging in orthodontics. Dentomaxillofacial Radiol 2011; 40: 24-34

4 De Cock J, Mermuys K, Goubau J et al. Cone-beam computed tomography: A new low dose, high resolution imaging technique of the wrist, presentation of three cases with technique. Skeletal Radiol 2012; 41: 93-96

5 Goerke S, Neubauer J, Zajonc H et al. Application Possibilities and Initial Experience with Digital Volume Tomography in Hand and Wrist Imaging. Handchirurgie Mikrochirurgie Plast Chir 2015; 47: 24-31

6 Kyriakou Y, Kolditz D, Langner 0 et al. Digital Volume Tomography (DVT) and Multislice Spiral CT (MSCT): an Objective Examination of Dose and Image Quality. Fortschr Röntgenstr 2011; 183: 144-153

7 Kalender WA, Kyriakou Y. Flat-detector computed tomography (FDCT). Eur Radiol 2007; 17: 2767-2779

8 Orth RC, Wallace MJ, Kuo MD. C-arm cone-beam CT: general principles and technical considerations for use in interventional radiology. J Vasc Interv Radiol 2008; 19: 814-820

9 Neubauer J, Voigt JM, Lang $H$ et al. Comparing the Image Quality of a Mobile Flat-Panel Computed Tomography and a Multidetector Computed Tomography. A Phantom Study Invest Radiol 2014; 49: 491-497

10 Damet J, Sans-Merce M, Miéville F et al. Comparison of organ doses and image quality between $\mathrm{CT}$ and flat panel XperCT scans in wrist and inner ear examinations. Radiat Prot Dosimetry 2010; 139: 164-168
11 Loubele M, Bogaerts R, Van Dijck E et al. Comparison between effective radiation dose of CBCT and MSCT scanners for dentomaxillofacial applications. Eur J Radiol 2009; 71: 461 - 468

12 Schulze $D$, Heiland $M$, Thurmann $H$ et al. Radiation exposure during midfacial imaging using 4- and 16-slice computed tomography, cone beam computed tomography systems and conventional radiography. Dentomaxillofacial Radiol 2004; 33: 83-86

13 Gosch D, Gosch K, Kahn T. Konversionsfaktoren zur Ermittlung der effektiven Dosis für Patienten aus dem Dosisflächenprodukt bei Röntgendurchleuchtungsuntersuchungen. Fortschr Röntgenstr 2007; 179: $1035-1042$

14 Menzel HG, Clement C, DeLuca P. ICRP Publication 110. Realistic reference phantoms: an ICRP/ICRU joint effort. A report of adult reference computational phantoms. Ann ICRP 2009; 39: 1-164

15 Rosslyn S. Digital Imaging and Communications in Medicine (DICOM) Part 14: Grayscale Standard Display Function. Medicine (Baltimore) 2004; 10: 3-4

16 Demehri S, Muhit A, Zbijewski W et al. Assessment of image quality in soft tissue and bone visualization tasks for a dedicated extremity conebeam CT system. Eur Radiol 2015; 25: $1742-1751$

17 Pearson $K$. Note on regression and inheritance in the case of two parents. Proc R Soc London 1895; 58: 240-242

18 Kendall MG. Rank correlation methods. C. Griffin; 1948

19 Bland JM, Altman DG. Multiple significance tests: the Bonferroni method. BMJ 1995; 310: 170

20 Stewart N, Gilula L. CT of the wrist: a tailored approach. Radiology 1992; 183: $13-20$

21 Chau ACM, Fung K. Comparison of radiation dose for implant imaging using conventional spiral tomography, computed tomography, and cone-beam computed tomography. Oral Surgery, Oral Med Oral Pathol Oral Radiol Endodontology 2009; 107: 559-565

22 Silva MAG, Wolf $U$, Heinicke F et al. Cone-beam computed tomography for routine orthodontic treatment planning: a radiation dose evaluation. Am J Orthod Dentofacial Orthop 2008; 133: 640.e1 - 640.e5

23 Neubauer J, Benndorf $M$, Lang $H$ et al. Comparison of Multidetector Computed Tomography and Flat-Panel Computed Tomography Regarding Visualization of Cortical Fractures, Cortical Defects. Medicine (Baltimore) 2015; 94: e1231

24 Hofmann E, Schmid M, Lell M et al. Cone beam computed tomography and low-dose multi- slice computed tomography in orthodontics and dentistry A comparative evaluation on image quality and radiation exposure Digitale dentale Volumentomografie und low-dose Mehrschicht-Computertomografie i. J Orofac Orthop 2014; 75: 384 - 398

25 Pauwels R, Beinsberger J, Stamatakis $H$ et al. Comparison of spatial and contrast resolution for cone-beam computed tomography scanners. Oral Surg Oral Med Oral Pathol Oral Radiol 2012; 114: 127-135

26 Schulze R, Heil U, Groß D et al. Artefacts in CBCT: A review. Dentomaxillofacial Radiol 2011; 40: $265-273$

27 Carrino JA, Al Muhit A, Zbijewski W et al. Dedicated cone-beam CT system for extremity imaging. Radiology 2014; 270: 816-824

28 De Vos W, Casselman J, Swennen GRJ. Cone-beam computerized tomography $(\mathrm{CBCT})$ imaging of the oral and maxillofacial region: A systematic review of the literature. Int J Oral Maxillofac Surg 2009; 38: 609625 